\title{
Fracture performance of high strength steels, aluminium and magnesium alloys during plastic deformation
}

\author{
Haiyan $\mathrm{Yu}^{\mathrm{a}}$ and You Wang \\ School of Automotive Engineering, Tongji University, 201804 Shanghai, China
}

\begin{abstract}
A series of uniaxial tension tests were performed for 5052 and 6061 aluminum alloys, AZ31B magnesium alloy, TRIP600 and DP600 steels, to obtain a better understanding of their fracture performance. Scanning electron microscope (SEM) observation of the microstructure evolution was conducted. The dimple structure, orientation relationship between the fracture surface and tensile direction, necking behavior were analyzed. The fracture mechanism and fracture mode of each material was discussed in detail. The results show that TRIP600 steel is subject to a typical inter-granular ductile fracture combined by shear fracture. DP600 steel belongs to mainly ductility mixed with normal fracture. Both 5052 and 6061 aluminum alloys are subject to a mixed ductility fracture and brittle fracture. AA5052 and AA6061 belong to a typical shear fracture and a normal fracture, respectively. Magnesium AZ31B is typical of a brittle fracture combined with normal fracture.
\end{abstract}

\section{Introduction}

As environmental problems become increasingly urgent, light weighting has been an indispensable aspect in the assessment of vehicle performance. Lightweight materials are more widely used in vehicles, such as high strength steels, aluminum and magnesium alloys. Their mechanical properties, manufacturability and service performance are the key issues during their application. Fracture has been reported to be one of the most common failure modes both in service and in manufacturing processes. High strength steels are more apt to fracture than low carbon steels because high strength is often related to low ductility $[1,2]$. Aluminum and magnesium alloys have also been reported to be more easily fractured than steels $[3,4]$ due to low strength and low elongation. Therefore, it is of vital importance to understand the fracture performance of these materials during plastic deformation.

Fracture mechanism and fracture mode are the two main assessment aspects for sheet metals. Srivatsan et al. [5] found that macroscopic fracture of AA7150 was by a shear mechanism, whereas microscopic fracture revealed both brittle and ductile failure mechanisms. Westermann et al. [6] investigated the influence of particles and solutes on the ductile fracture of four different aluminum alloys in as-cast and homogenized conditions. Hopperstad et al. [7], Narasayya et al. [8], Chen [9] and Xing et al. [10] studied the fracture behavior of some aerospace aluminum alloys, such as 7075, 2219

\footnotetext{
${ }^{\text {a }}$ Corresponding author: yuhaiyan@tongji.edu.cn
}

This is an Open Access article distributed under the terms of the Creative Commons Attribution License 4.0, which permits unrestricted use, distribution, and reproduction in any medium, provided the original work is properly cited. 


\section{MATEC Web of Conferences}

Table 1. Chemical compositions of the investigated materials (weight \%).

\begin{tabular}{cccccccccc}
\hline Materials & Thickness $(\mathrm{mm})$ & $\mathrm{Al}$ & $\mathrm{Mg}$ & $\mathrm{Fe}$ & $\mathrm{Si}$ & $\mathrm{Mn}$ & $\mathrm{Cu}$ & $\mathrm{Zn}$ & $\mathrm{C}$ \\
\hline AA5052 & 1.0 & 94.92 & 3.41 & 0.49 & 0.25 & 0.10 & 0.1 & 0.10 & 0.07 \\
AA6061 & 2.4 & Balance & 0.9 & 0.7 & 0.7 & 0.15 & 0.2 & 0.25 & $\mathrm{DU}$ \\
AZ31B & 1.0 & 3.17 & 95.54 & 0.005 & 0.1 & 0.50 & 0.01 & 0.86 & $\mathrm{DU}$ \\
TRIP600 & 1.0 & 0.027 & DU & Balance & 1.54 & 1.47 & 0.016 & $\mathrm{DU}$ & 0.11 \\
DP600 & 1.2 & 0.039 & DU & Balance & 0.013 & 1.76 & 0.04 & DU & 0.086 \\
\hline
\end{tabular}

and 7085. Wei et al. [11] compared the deformation and fracture behavior of 1100, 6061 and 2A12 aluminum alloy bars. They concluded that AA1100 belongs to ductile fracture and AA6061 suffers a mix of ductile fracture and brittle fracture, whereas $2 \mathrm{~A} 12$ is subject to a typical brittle fracture. For magnesium alloys, Zheng [12] and Zhang [13] investigated the fatigue crack growth and failure behavior of AZ31B sheets. Tari [14] and Yooh [15] demonstrated that the fracture mechanism of AZ31B sheets varied with the temperature.

Transformation-induced plasticity (TRIP) steels and dual-phase (DP) steels are two valuable high strength steels that can be stamped at room temperature. Their damage mechanism and failure behavior are very complex and strongly affected by so many aspects. Uthaisangsuk [16] concluded that the fracture mode of TRIP and DP steels are dependent on the stress state or triaxiality, the purity degree, the volume fraction of retained austenite, the carbon content of retained austenite and martensite as well as the locations of the neighboring austenite grain and martensitic islands. Yang [17] analyzed the primary and secondary fracture surfaces of DP590 steel. The results showed that the secondary fracture is cleavage, which hinted a brittle fracture. Feng [18] found the overall fracture mode of DP steel is determined by the competition between the brittle fracture of the martensite and ductile damage in the ferrite under multi-axial loading conditions.

In this study, the fracture mechanism and fracture mode were investigated to gain a better understanding of TRIP600, DP600, AZ31B magnesium alloys, and 5052 and 6061 aluminum alloys. A series of uniaxial tension tests were performed. Optical and SEM observations on the fracture surfaces were conducted. Micro-characteristics of the fracture surfaces were discussed, and the fracture mechanism of each material was analyzed. The orientation relationship between the fracture surface and the acting force and necking deformation near the fracture surface were also examined.

\section{Experiments}

\subsection{Materials}

In this study, five commercial sheets, including AA5052 and AA6061 aluminum alloys, AZ31B wrought magnesium alloy, and TRIP600 and DP600, steels were studied. All of the materials were made in China. The detail information on the thickness and chemical compositions were listed in Table 1.

\subsection{Uniaxial tension}

A series of uniaxial tension tests at room temperature and a nominal strain rate of $2 \times 10^{-3} / \mathrm{s}$ were performed on dog-bone sheet specimens of the five sheets. The specimens were prepared according to the ASTM E8/E8M-09. The uniaxial tension tests were conducted in a Zwick universal testing machine and an extensometer with $50 \mathrm{~mm}$ gauge was used.

\subsection{Optical and SEM observations}

To examine the microstructures of the five sheets, optical microscopy machine observation was performed. Aluminum samples were eroded in Keller's reagent [19]. Magnesium AZ31B samples were 

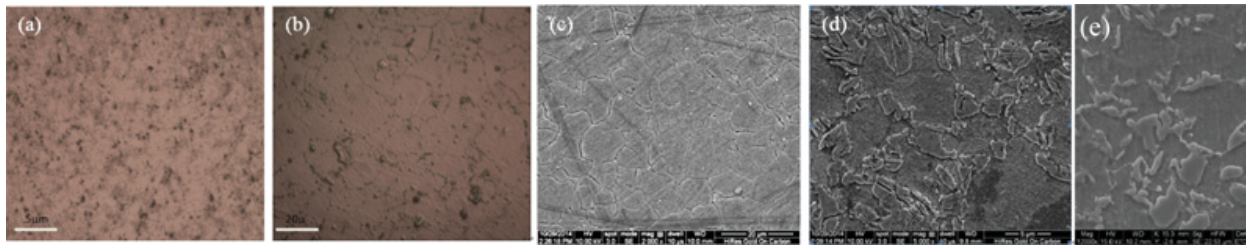

Figure 1. Microstructures of the investigated materials in the as-received condition. (a) AA5052, (b) AA6061, (c) AZ31B, (d) DP600 and (e) TRIP600.

etched using an acetic picral solution composed of picric acid, acetic acid, ethanol and water [20]. Before SEM observation, the AZ31B samples were treated with conductive coating by gold sputtering to enhance the conductivity of the specimen slice.

From the fractured specimens, fracture surfaces approximately $10 \mathrm{~mm}$ long were cut, and then they were examined using a QUANTA FEG250 scanning electron microscopy (SEM) instrument.

\section{Results and discussion}

\subsection{Initial microstructure}

Figures 1(a) and 1(b) show the microscope images of AA5052 and AA6061, respectively. Due to the solution strength mechanism, they have large grains. The secondary $\mathrm{Mg}_{2} \mathrm{Si}$ particles can be observed. Because AA6061 has more silicon than AA5052, more $\mathrm{Mg}_{2} \mathrm{Si}$ can be observed among the grains boundaries, as shown in Fig. 1(b). In addition, AA6061 has finer precipitates of a uniform size, whereas AA5052 has coarser ones of varying sizes, which can also lead to different strengths between the two materials. Therefore, AA6061 has a higher strength than AA5052. Figure 1(c) shows the optical microscope image of the $\mathrm{AZ31B}$ sheet. The equiaxial grains were coarse and $\mathrm{Mg}_{1} 7 \mathrm{Al}_{1} 2$ intermetallic island compounds were observed among the grain boundaries. These compounds exist as the secondary particles, and they are harmful to the ductility of the material.

Figures 1(d) and 1(e) show the SEM images of the DP600 and TRIP600 sheets, respectively. The DP600 steel consists of ferrites and martensites. The bright white island-like martensites distribute among the gray ferrite matrix. Moreover, the edges of the martensite islands are bright white, whereas the island kernels are gray. This is because the manganese $(\mathrm{Mn})$ element concentrates on the boundary of the martensite islands when it spreads along the grains. These circles of high Mn content can be more easily quenched than the kernels. In Fig. 1(e), the equiaxial polygon structures are ferrites. The white structures are retained austenites and martensites, and the black structures existing in the grain boundaries are bainites. Some amount of retained austenites can transform to martensites under plastic forming at room temperature. The martensitic transformation can enhance the overall work hardenability of TRIP steel. Therefore, TRIP steels combine high strength and excellent ductility.

\subsection{Engineering stress-strain curves}

Figure 2 shows the engineering stress-strain curves of the five sheets. TRIP600 and DP600 steels have similar tensile strengths but different elongation. DP600 has a higher initial working hardenability, whereas TRIP600 can keep its high working hardenability over a larger strain range. Compared with TRIP600 and DP600 steels, the two aluminum alloys and AZ31B magnesium alloy have far lower strength and lower elongation. 


\section{MATEC Web of Conferences}

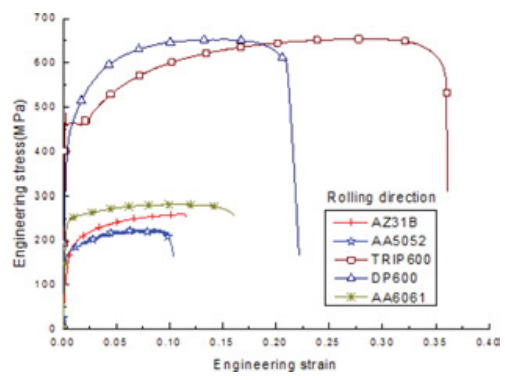

Figure 2. Engineering stress-strain curves of the tested materials.
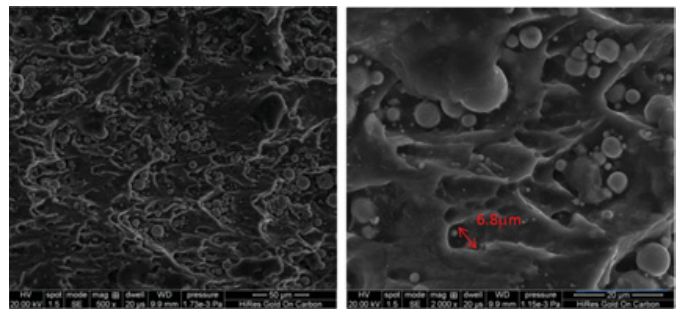

Figure 3. Fracture micrograph of AA5052.
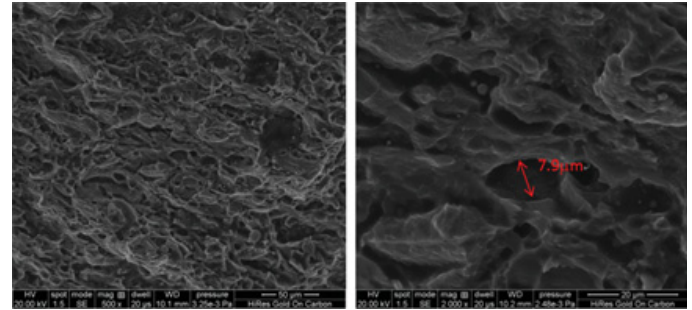

Figure 4. Fracture micrograph of AA6061.

\subsection{Fracture surfaces}

Figures 3-7 show the fracture surfaces observed under SEM for the AA5052, AA6061, AZ31B, TRIP600 and DP600 sheets. For each material, the dimple structures were observed in different magnitudes. From Fig. 4, one can see there are two types of dimples, large and deep dimples and small dimples containing some spherical particles. The former type of dimples indicate a ductile fracture for the AA5052, whereas the latter dimples indicate a brittle fracture. There are a large number of spherical particles distributing among the wall and bottom of the small dimples. These particles demonstrate that the material has suffered an inter-granular fracture, and the particles are the products of the fractured brittle precipitates. Therefore, the fracture mechanism of AA5052 is a mixture of ductile fracture and brittle fracture.

Compared with Fig. 3, Fig. 4 shows smaller voids rather than parabolic dimples. These voids distribute disorderly. Most of the dimples are $7 \sim 8 \mu \mathrm{m}$ wide and $10 \sim 15 \mu \mathrm{m}$ long. Moreover, the overall fracture surface seems to be vertical with the tension direction, whereas a slanted fracture surface is obvious in Fig. 3. This shows that the normal direction of the fracture surface of AA6061 is vertical with the first principle stress under uniaxial tension. So the fracture mechanism of AA6061 is mainly brittle fracture.

Figure 5 shows the fracture surface of the AZ31B alloy. There are many typical flat surfaces with cleavage-like patterns, and the edges are sharp and bright without any hints of necking before fracturing. Furthermore, on the bottom of the flat surfaces some shallow small dimples and a few cracked particles can be observed. These are symptoms of brittle fracture. Therefore, the fracture mechanism of the studied AZ31B alloy is brittle fracture.

Figure 6 shows the fracture surface of the TRIP600 steel. The parabolic dimples are fine and densely distributed, and their size is smaller than that in the other four alloys. This can be one of the explanations why TRIP600 steels combine higher strength and better ductility than those materials. Moreover, from the high magnification SEM image, one can see that the edges of the dimples are smoother and duller 

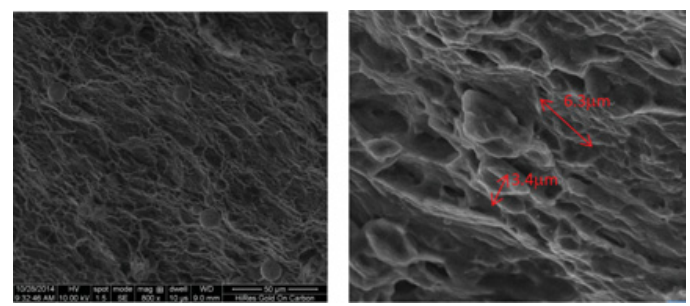

Figure 5. Fracture micrograph of AZ31B magnesium sheet observed by SEM.

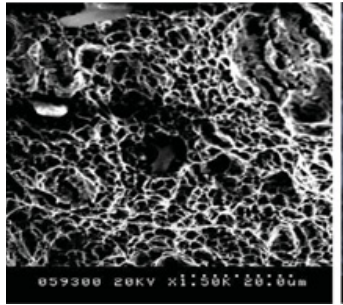

Figure 6. Fracture micrograph of the TRIP600 steel.

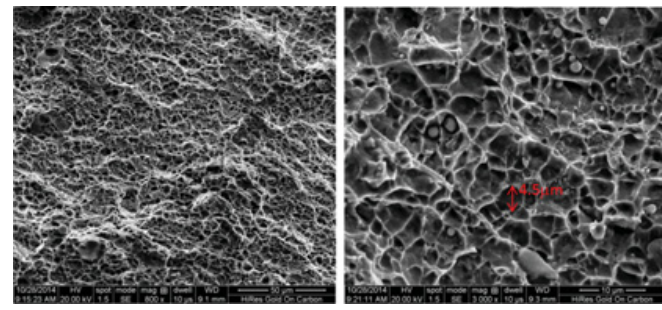

Figure 7. Fracture micrograph of the DP600 steel.

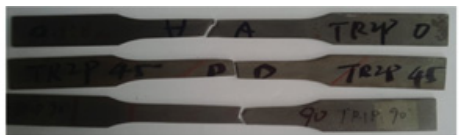

(a) TRIP600

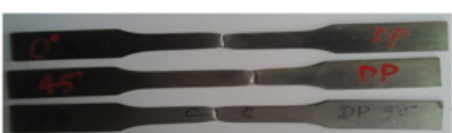

(b) DP600

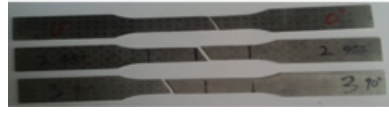

(c) AA5052

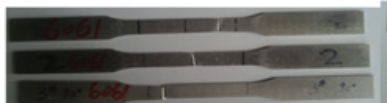

(d) AA6061

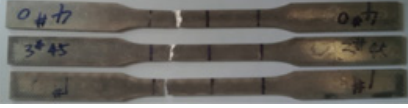

(e) AZ31B

Figure 8. Orientation relation between the fracture surface and the tension direction.

than those in Fig. 3 and Fig. 4. This indicates that large plastic deformation occurred before fracturing, and the cracks develop within the grains. The cracks grow and intersect mainly by trans-granular fracture. No obvious cleavage-like surfaces and small particles are observed. Therefore, the fracture mechanism of TRIP600 steel is a typical ductile fracture.

Figure 7 shows the fracture micrograph of the DP600 sheet. From the left low magnification image, one can see that the dimples are fine and uniformly distributed, which suggests a ductile fracture mechanism. In the right image with high magnification, there are many small particles located at the bottom of the dimples. Moreover, the edges of the dimples are bright white. The depth of the dimples is smaller than that for TRIP600 shown in Fig. 6. These characteristics indicate brittle fracture for the DP600 sheet. Therefore, the tested DP600 steel is subject to a mixture of ductile fracture and brittle fracture, with ductile fracture being dominant.

Figures 8(a)-(e) show the fractured specimens tested in uniaxial tension for TRIP600, DP600, AA5052, AA6061 and AZ31B magnesium alloy, respectively. In each image, the three specimens were cut along the angles of $0^{\circ}, 45^{\circ}$ and $90^{\circ}$ to the rolling direction. Figure 8 (a) shows that the fracture surface of the TRIP600 steel is subjected to mainly shear fracture because the fracture surface obliquely intersects the tension direction. In Fig. 9(b), the fracture surface of the DP600 steel is almost vertical to 


\section{MATEC Web of Conferences}

the tension direction, which indicated a normal fracture. AA5052 alloy (see Fig. 8(c)) is also subject to typical shear fracture. AA6061 (see Fig. 8(d)) and AZ31B (see Fig. 8(e)) are subject to normal fracture.

From the aspect of the necking behavior of these materials before fracture, both TRIP600 and DP600 steels exhibited eminent necking behavior because the width near the fracture surface is significantly reduced. Little width reduction can be observed near the fracture surfaces of AA5052 and AA6061. Figure 8(e) shows that neither the width nor the thickness displayed any hints of necking. This revealed a brittle fracture mechanism for the tested AZ31B magnesium alloy.

\section{Conclusions}

A series of uniaxial tension tests for TRIP600 and DP600 steels, AZ31B magnesium alloy, and 5052 and 6061 aluminum alloys were performed. Their fracture mechanism and fracture mode were discussed from the micrograph, the orientation and the necking behavior of the fracture surfaces. Several conclusions were obtained.

(1) TRIP600 steel undergoes typical ductile fracture combined by shear fracture. DP600 steel belongs to mainly ductile mixed with normal fracture.

(2) Both 5052 and 6061 aluminum alloys are subject to a mixed ductile fracture and brittle fracture; ductile fracture plays the main role for the AA5052, whereas brittle fracture plays a major role for the AA6061. AA5052 is subject to typical shear fracture, and AA6062 suffers from normal fracture based on the orientation relation of the fracture surfaces.

(3) The fracture mechanism of AZ31B magnesium alloy is mainly brittle fracture combined with normal fracture.

This project was supported by the National Natural Science Foundation of China under grant No. 51175382.

\section{References}

[1] Wurong Wang, Changwei He, Zhonghua Zhao, Xicheng Wei, Mater. Des. 32(6), 3320 (2011)

[2] Y. Abe, T. Ohmi, K. Mori, T. Masuda, J. Mater. Process. Technol. 214(9), 1838 (2014)

[3] Xiaolei Cui, Xiaosong Wang, Shijian Yuan, Int. J. Mech. Sci. 88, 232 (2014)

[4] Liwei Lu, Tianmo Liu, Yong Chen, Zhongchang Wang, Mater. Charact. 67, 93 (2012)

[5] T.S. Srivatsan, G. Guruprasad, V.K. Vasudevan, Mater. Des. 29(4), 742 (2008)

[6] I. Westermann, K.O. Pedersen, T. Furu, T. Børvik, O.S. Hopperstad, Mech. Mater. 79, 58 (2014)

[7] O.S. Hopperstad, T. Børvik, M. Fourmeau, K.O. Pedersen, A. Benallal, Procedia Materials Science, 3, 51 (2014)

[8] Ch.V.A. Narasayya, P. Rambabu, M.K. Mohan, R. Mitra, N.E. Prasad, Procedia Materials Science, 6, 322 (2014)

[9] Songyi Chen, Kanghua Chen, Pen-xuan Dong, Shengping Y.E., Lanping Huang, Trans. Nonferrous Met. Soc. China, 24(7), 2320 (2014)

[10] Mingzhi Xing, Yonggang Wang, Zhaoxiu Jiang, Def. Technol. 9(4), 193 (2013)

[11] Gang Wei, Wei Zhang, Wei Huang, Nan Ye, Yubo Gao, Yugang Ni, Int. J. Impact Eng, 73, 75 (2014)

[12] Sanlong Zheng, Qin Yu, Zengliang Gao, Yanyao Jiang, Eng. Fract. Mech. 114, 42 (2013)

[13] Hongxia Zhang, Wenxian Wang, Yinghui Wei, Jinyong Li, Jianling Wang, Trans. Nonferrous Met. Soc. China, 21(6), 1225 (2011)

[14] D.G. Tari, M.J. Worswick, S. Winkler. J. Mater. Process. Technol. 213(8), 1337 (2013)

[15] J. Yoon, Y. Lee. Mater. Des. 55, 43 (2014) 
[16] V. Uthaisangsuk, U. Prahl, W. Bleck. Eng. Fract. Mech. 78(3), 469 (2011)

[17] Min Yang, Y.J. Chao, Xiaodong Li, D. Immel, Jinzhu Tan, Mater. Sci. Eng., A, 497(1-2), 462 (2008)

[18] N. Vajragupta, V. Uthaisangsuk, B. Schmaling, S. Münstermann, A. Hartmaier, W. Bleck, Comput. Mater. Sci. 54, 271 (2012)

[19] M.V. Farahani, E. Emadoddin, M. Emamy, A.H. Raouf, Mater. Des. 54, 361 (2014)

[20] Weiqin Tang, Shiyao Huang, Dayong Li, Yinghong Peng, J. Mater. Process. Technol. 215, 320 (2015) 